\title{
Respirable Dust Monitoring in Construction Sites and Visualization in Building Information Modeling Using Real-time Sensor Data
}

\author{
Nour Smaoui, ${ }^{1}$ Kyungki Kim, ${ }^{2}$ Omprakash Gnawali, ${ }^{1}$ \\ Young-Joo Lee, ${ }^{3}$ and Wonho Suh ${ }^{4 *}$ \\ ${ }^{1}$ Department of Computer Science, University of Houston, \\ 551 Cullen Blvd., Room 501 Houston, TX 77204, USA \\ ${ }^{2}$ Department of Construction Management, University of Houston, \\ 4734 Calhoun Road \#111, Houston, TX 77204, USA \\ ${ }^{3}$ School of Urban and Environmental Engineering, Ulsan National Institute of Science and Technology (UNIST), \\ 50 UNIST-gil, Eonyang-eup, Ulju-gun, Ulsan 44919, South Korea \\ ${ }^{4}$ Department of Transportation and Logistics Engineering, Hanyang University ERICA Campus, \\ 55 Hanyangdaehak-Ro, Sangnok-Gu, Ansan 15588, South Korea
}

(Received April 5, 2017; accepted February 26, 2018)

Keywords: dust monitoring, sensor data, BIM, real-time monitoring, construction site monitoring

Construction activities, involving cutting, drilling, and grinding of materials, often produce toxic respirable dust that can cause fatal diseases and illnesses. To protect workers from breathing excessive amounts of respirable dust at job sites, superintendents should continuously monitor the level of respirable dust in workspaces and make timely interventions for overexposed workers. However, current practices of respirable dust monitoring have critical drawbacks, and superintendents cannot accurately estimate workers' exposures to respirable dust or make prompt decisions to protect the workers. Therefore, there is a need for real-time air dust monitoring that can be deployed ubiquitously at a construction site and be integrated as part of daily construction management. In this research, we developed a real-time dust monitoring system that comprises a network of low-cost mobile dust sensors and visualization in building information modeling (BIM). Single-board computers and dust sensors were integrated as field deployment units. Inaccurate sensors were calibrated automatically on the basis of an accurate ground truth sensor. A BIM-based visualization system was developed to present the data collected from dust sensors in real time. A prototype system was developed and tested in a controlled environment.

\section{Introduction}

Approximately 2.3 million workers are exposed to dust in their workplaces. In particular, 2 million construction workers are frequently exposed to the risk of breathing dust when they drill, cut, crush, or grind silica-containing materials such as concrete and stone. ${ }^{(1)}$

*Corresponding author: e-mail: wonhosuh@hanyang.ac.kr http://dx.doi.org/10.18494/SAM.2018.1871 
In fact, more deaths occurred in construction than any other industry in the U.S. due to workers breathing respirable dust containing silica. ${ }^{(2)}$ However, current practices in the construction industry have not been effective in minimizing/preventing dust exposure and providing workers with healthy working conditions. Construction managers or employers are required to use engineering controls (such as water or ventilation) or respirators if engineering controls are not applicable. Construction managers should also limit all other workers from entering high-exposure areas and provide medical exams to highly exposed workers according to the monitoring results. Despite the important roles of construction managers, current practices of air dust monitoring have critical drawbacks that limit the managers' ability to minimize the hazardous dust exposures in their daily construction management activities.

First, current practices of air dust monitoring are mostly based on the postexposure analysis of air dust samples. Air dust samples collected from workplaces require laboratory analysis that takes multiple days to weeks. Owing to the time gap between the exposure and analysis, air dust sampling cannot be used to assist construction managers in making decisions immediately to protect workers. Therefore, advanced air dust monitoring is needed to provide an immediate estimate of exposures.

Second, workers, times, and locations in current air dust monitoring are limited. The circumstances at a construction project are complex and change continuously, but air dust monitoring is conducted sparsely for specific workers, times, and locations. In this way, construction managers may have a very limited insight about how workers are exposed to hazardous dust during construction. To overcome this limitation, air dust monitoring tools should be easily deployable to multiple workers and locations during the entire construction period.

Finally, current practices of air dust monitoring are separated from construction management processes and tools that are popular in the field. Commonly used construction management tools, such as building information modeling (BIM) and construction schedules, are not used to facilitate environmental monitoring and control. ${ }^{(2,3)}$ As a result, even existing databases on dust exposure cannot be used by contractors with ease. Many of these databases are unreliable owing to the lack of details about construction site circumstances and diverse ways of collecting, analyzing, and recording the samples within a database. Air dust monitoring should be incorporated into construction management and visualization tools so that it can be easily conducted as an essential part of daily construction management.

Therefore, there is a strong need to identify a real-time air dust monitoring system that can be deployed ubiquitously in a construction project and managed as part of daily construction management. An appropriate solution for this can be BIM, which serves as real-time 3D visualization and as a communication tool that is more accessible and that has the ability to analyze risk and landscape modeling, and to exchange information between team/project members. BIM is acknowledged by the construction industry as a possible tool that may improve worker safety and health. For continuous monitoring and exposure estimation of individual workers, this project proposes a solution consisting of (1) real-time dust monitoring using low-cost dust sensors, (2) worker location tracking, and (3) integration with BIM for 
visualization and communication. The limitations of existing technologies and opportunities are also discussed.

\section{Related Work}

Riaz et al. used wireless sensors to monitor confined workspaces, integrating BIM for visualizations of the collected data. ${ }^{(4)}$ The use of wireless sensor networks (WSNs) and BIM is repeated in this and many other research studies, showing the high efficiency of BIM and sensors in health and safety plans. In this study, we monitored oxygen and temperature extremes, which contribute to making work environments hazardous. Positive industry feedback from their results shows that real-time sensing and visualization can facilitate intelligent and timely decision making to protect workers against unhealthy conditions.

Cheung et al. presented a web-based system for monitoring and assessing construction safety and health performance, called the construction safety and health monitoring (CSHM) system. ${ }^{(5)}$ In their design, they combined internet and database systems to create a comprehensive safety and health management tool. The conceptual framework of their research has: (a) web-based interfaces (templates), (b) knowledge base, (c) output data, and (d) benchmark group. Their research emphasizes gathering knowledge base data such as rules, guidelines, and best practices in one easily accessible database and combining this data with field output data to come up with a benchmark with which to share and compare performance results. Designing a template of relevant data (related to safety and health performance) is the key of their research as it leads to a useful prioritization of numerous possible paramaters. In their study, the parameters adopted are mainly derived from two sources: (a) an intensive literature review, followed by (b) interviews with experts in the field. The focus of their research is data submitted by employees and managers but their model can combine data input from sensors with input from superintendents and project managers to give a more accurate assessment of site conditions. Many studies attempted to improve worker health and safety by collecting jobsite data in real time and utilizing the data for decision making. However, the monitoring of respirable dust has not been achieved despite the critical impact on health.

Related to dust level monitoring, the US Environmental Protection Agency (EPA) classified low-cost dust sensors as those below $\$ 2500$ in their dust sensor evaluation. ${ }^{(6)}$ However, these sensors costing thousands of dollars are not suitable for deployment in large construction sites. For deployment around large construction sites with hundreds of workers, sensors of even lower cost should be used. Our preliminary sensor tests found that their accuracy is lower than that of expensive monitoring tools. Also, dust composition cannot be identified without laboratory tests. Multiple locations in a large construction site can be monitored economically during the entire construction if the performance of the low-cost sensors can be improved. A research study ${ }^{(7)}$ attempted to improve the accuracy by automatically calibrating sensors based on a ground truth sensor mounted on a self-navigating robot. In real-time monitoring, worker location information is essential to accurately estimate the exposures of individual workers to safety and health risk sources. ${ }^{(8,9)}$ Even though many approaches in the past measured the respirable dust levels in specific locations, ${ }^{(10,11)}$ none could measure exposures to individual 
workers. Tracking a worker requires a reliable location sensing technology. GPS-based localization does not work well in indoor and construction environments ${ }^{(12,13)}$ despite many improvements proposed in the literature. ${ }^{(14)}$ Researchers have explored the use of various technologies, including fingerprinting of wireless signals, ${ }^{(15)}$ information fusion from different sources, ${ }^{(16,17)}$ utilizing other modalities to improve accuracy, ${ }^{(17,18)}$ ultrasonic ranging, ${ }^{(19,20)}$ and radio-frequency identification (RFID) tags. ${ }^{(21)}$ Ultrawide-band (UWB)-based location tracking is considered the most promising technology ${ }^{(22)}$ and has been actively studied to improve its accuracy. ${ }^{(23-25)}$ In this research, we use UWB-based tracking and further adapt it for use at construction sites.

In a construction setting with obstructions and an indoor environment, tracking of multiple objects (workers) at a site is challenging because GPS does not work well. UWB-based tags are most promising for indoor people-location tracking but still suffer from inaccuracies according to our preliminary experiments with state-of-the-art UWB tracker tags. Awareness about worker location can enable a more accurate estimation of workers' exposure to respirable dust. The development of UWB anchor placement guidelines based on empirical studies and data fusion including context information should allow us to improve the accuracy of UWB location trackers sufficiently so that they can be used on a large scale in a construction environment. BIM is a construction management tool widely used in the construction industry, mainly to visualize buildings and analyze construction plans manually ${ }^{(26)}$ or automatically. ${ }^{(27)}$ Environmental information has not been incorporated extensively in BIM. Developing customized dashboards and visual summaries is a technically feasible way of helping construction superintendents to better use the results of real-time dust monitoring.

\section{Real-time Dust Monitoring and Worker Location Tracking for Exposure Estimation}

The objective of this research is to propose a framework for real-time dust monitoring and visualization in BIM. We have developed a real-time dust monitoring system comprising a network of low-cost mobile dust sensors and visualization in BIM. Single-board computers and dust sensors were integrated as field deployment units. A BIM-based visualization system was developed to present the data collected from dust sensors in real time. A prototype system was developed and tested in a real-world construction project.

The proposed framework is illustrated in Fig. 1. This project overcomes the temporal and spatial deficiency of conventional dust monitoring by integrating real-time worker location tracking and dust monitoring. The acquisition of real-time dust levels and locations of individual workers in the entire construction site enables the continuous exposure monitoring of individual workers.

In pretask dust sensor calibration, low-cost dust sensors were calibrated automatically at a designated location in a construction site. Calibration may be needed daily or weekly depending on the results of this project. After calibration, the performances of individual sensors were optimized using a high-precision sensor. In this way, a construction site needs a maximum of one expensive high-precision sensor. 

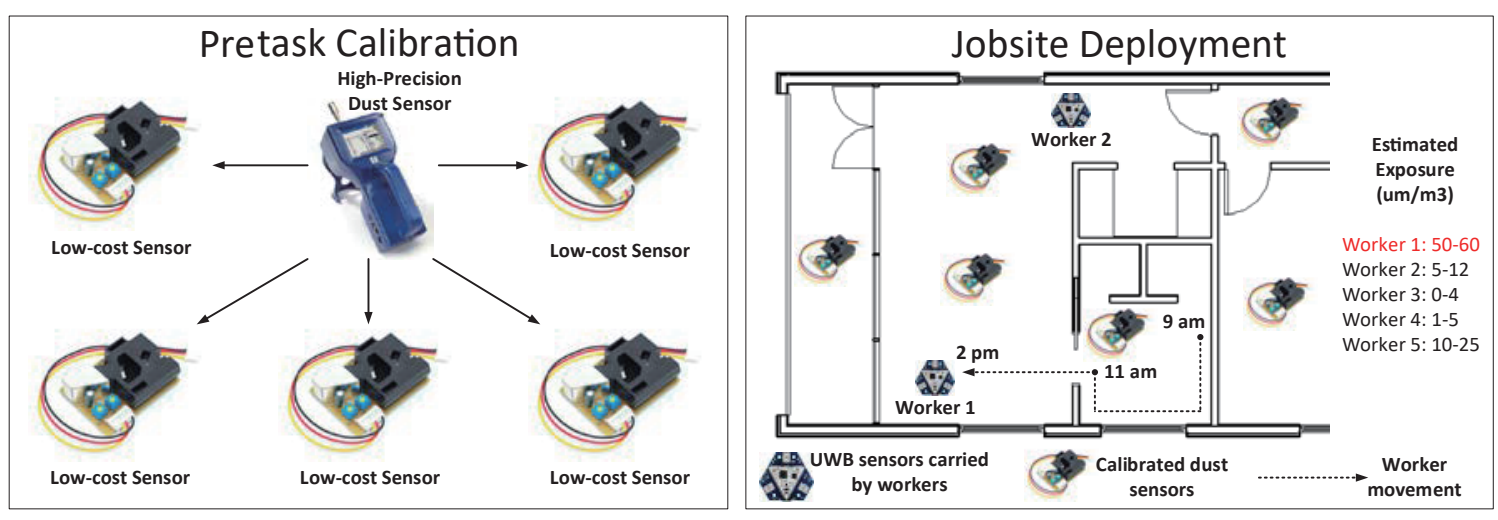

Fig. 1. (Color online) Framework of dust monitoring and location tracking.

When construction starts, low-cost dust sensors and location tracking tags are deployed. Dust sensors are located in dusty workspaces and tracking tags are attached to all the workers and managers in a construction site. On the basis of the data collected from sensors, total respirable dust exposures by individual workers are calculated.

\section{Implementation and Data Collection}

A prototype system was developed and tested in a controlled environment as shown in Fig. 2. In pretask dust sensor calibration, low-cost dust sensors such as PMS5003 and PMS7003 may display erroneous behavior when they measure dust. To build a reliable system that reports dust sensing information, these devices must be calibrated to report the actual ground truth data. For this purpose, we relied on a high-end dust sensor such as TSI DustTrack II to report the ground truth data. The calibration is performed by collecting dust measurements using PMS7003 and the ground truth sensor at the same time in a calibration chamber. We used a box as a calibration chamber to reduce the risks of airflow variation and to ensure a uniform distribution of dust sprayed around the sensors. During the calibration, we spray dust in the box every $3 \mathrm{~min}$ for $30 \mathrm{~min}$. In every spraying iteration, we used a dust sprayer to perform three consecutive sprays. Data was collected using PMS7003 sensors and the ground truth sensor every second. The pretask dust sensor calibration process is shown in Fig. 3.

Once the data collection was done, we analyzed the raw data. Our first observation was that the amount of noise was considerable and we could not find a pattern. Therefore, we used a moving average to smooth the data and remove the noise. As Fig. 4(a) shows, we applied a fast Fourier transform to the originally collected data and to the smoothed data to extract the signal frequencies related to dust collection and noise. We used multiple moving average window sizes to find the best window that reduced the noise and preserved a reasonable amount of useful data. We found that a window size of 20 was sufficient to reduce a considerable amount of noise and preserve an appropriate amount of useful data as shown by Fig. 4(b). We noticed from the smoothed data that we could use a linear function to calibrate the sensors. We used half of the collected data to train the linear function and the other half to validate the calibration. 


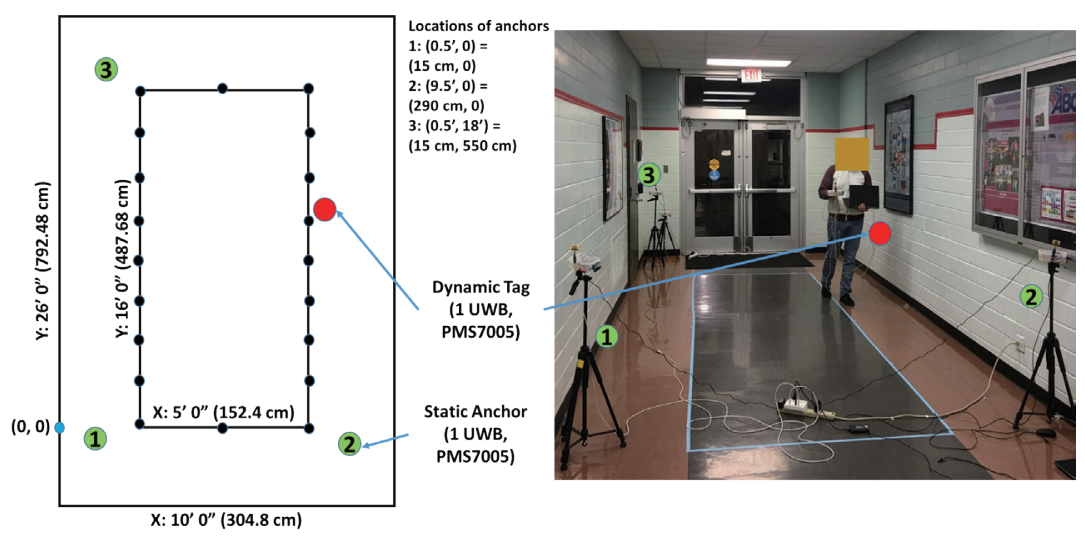

Fig. 2. (Color online) Dust sensing and location tracking experimental setup.

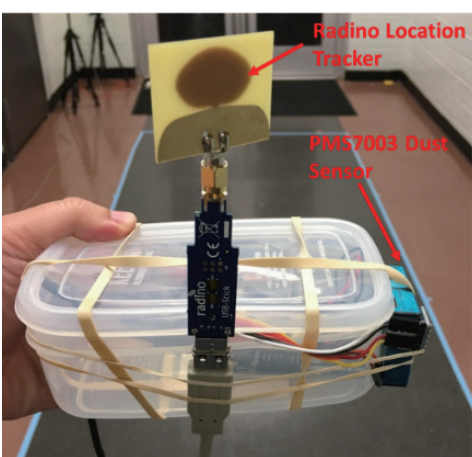

(a)

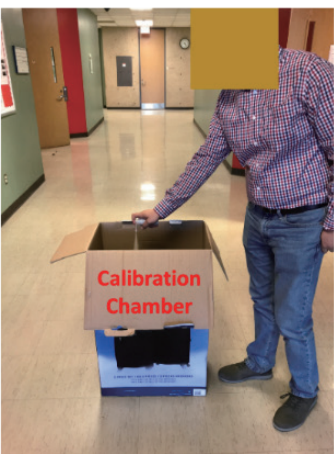

(b)

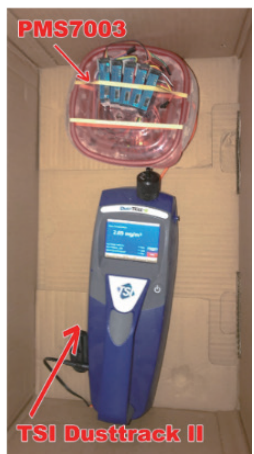

(c)

Fig. 3. (Color online) (a) A unit with location and dust sensors, (b) dust sensor calibration, and (c) inside the dust calibration chamber.

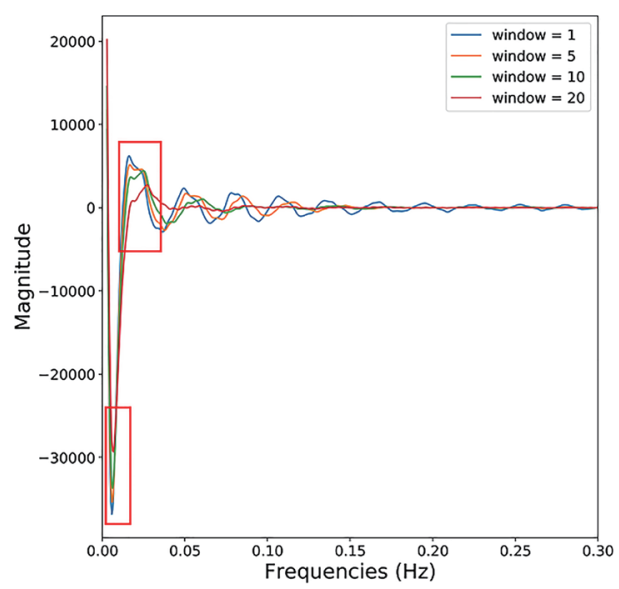

(a)

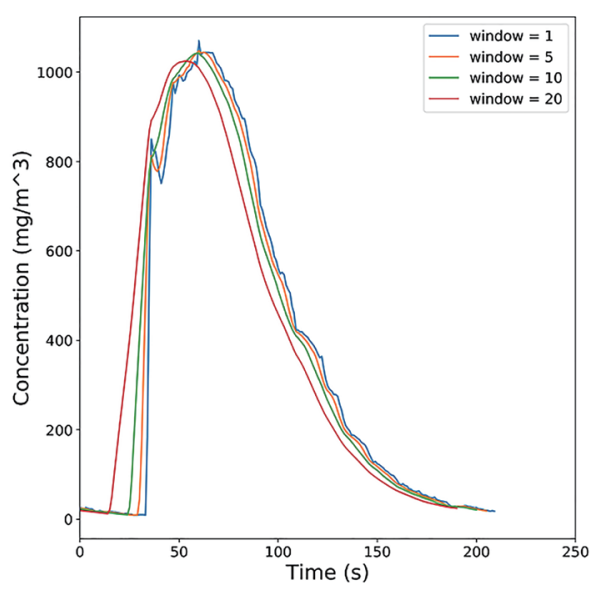

(b)

Fig. 4. (Color online) Comparison between different moving average window sizes and their smoothing effect on the noisy data: (a) Fast Fourier transform of smoothed sensor data. (b) Smoothed sensor data. 
Although the results were convincing, we investigated the use of higher order polynomials to do the data fitting. For every sensor, we tried 10 polynomial fitting functions with orders varying between 1 and 10. Then, based on the average error between the ground truth and the fitted data, we defined the best fitting function order for every sensor.

For worker location tracking, UWB was chosen as an RF communication technology using frequencies between 3 and $10 \mathrm{GHz}$ with bandwidths exceeding $500 \mathrm{MHz}$, therefore allowing very fine time resolution on the order of picoseconds that could be converted spatially to the centimeter level. For our experiments, we used a radio node equipped with Decawave DW1000 UWB chips and running the double-sided two-way ranging (DS-TWR) protocol as shown in Fig. 5. There are two types of node in our systems: (1) anchors, i.e., spatially static nodes that provide a reference for localization, and (2) tags, i.e., spatially mobile nodes that are localized.

Both types of nodes ran the DS-TWR protocol. This protocol has two phases: (1) initialization phase: this is their discovery; the tags send a "Blink" message in broadcast to inform the surrounding anchors of their existence; then the anchors reply to the detected tag with a "Init ranging" message to finish the discovery process, and (2) ranging phase: in this phase, the DS-TWR protocol is applied. The tag initiates a communication with three of the detected anchors by sending a "Poll" message to them, specifying the order in which they need to reply and the timestamp of the transmission of the "Poll" message. The anchors reply in the designated order to the tag with a "Poll Ack" message that contains the timestamp of reception of the "Poll" message and the timestamp of the transmission of the "Poll Ack" message. At this point, the tag replies to the anchors with a "Range" message containing the timestamp of

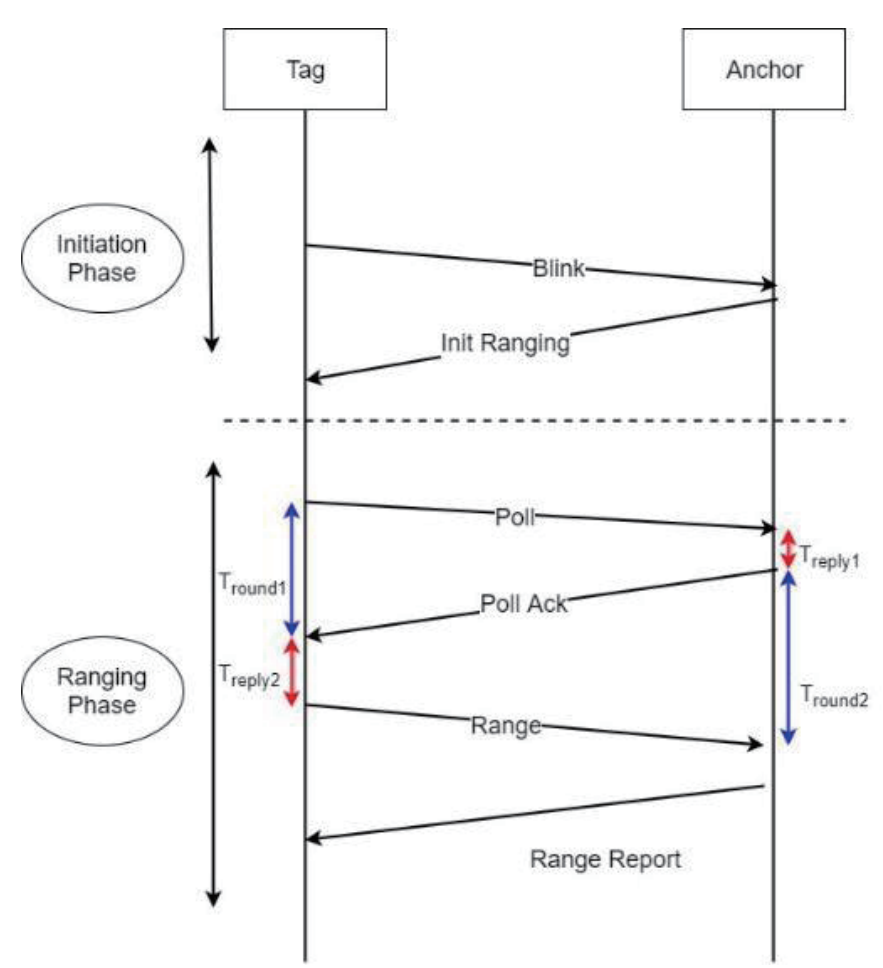

Fig. 5. (Color online) Ranging protocol. 
"Poll Ack" reception and the timestamp of transmission of "Range" message. All the anchors are, therefore, capable of calculating the time-of-flight between them and the tag by applying the formula (formula for ranging) and can generate the range value. This value is communicated to the tag with a "Range report" message.

$$
\hat{T} \text { prop }=\frac{\text { Tround } 1 \times \text { Tround } 2-\text { Treply } 1 \times \text { Treply } 2)}{(\text { Tround } 1+\text { Tround } 2+\text { Treply } 1+\text { Treply } 2)}
$$

\section{Results}

In this section, we evaluate our system in terms of three aspects related to dust sensor calibration, location tracking, and spatial inference.

\subsection{Dust sensor calibration}

In this experiment, five PMS 7003 dust sensors were calibrated on the basis of the values from a ground truth sensor (TSI DustTrak II). As a result, the dust concentrations of the lowcost PMS 7003 were fitted according to more accurate values. As shown in Figs. 6(a) and 6(b), PMS 7003 sensors were reporting significantly greater values than the ground truth sensor.

The possible reasons for these gaps may include fans of dust sensors that are not properly calibrated or the irregular distribution of dust in the chamber. In this experiment, the former is considered to be the main reason. As shown in Fig. 6(c), the dust concentrations of low-cost sensors were adjusted properly. The accuracy of our calibration method is shown in Fig. 6(d), where the maximum concentration error with regards to the ground truth sensor is around 6 $\mathrm{mg} / \mathrm{m}^{3}$. Given that errors of more than $2000 \mathrm{mg} / \mathrm{m}^{3}$ were observed before calibration, it can be concluded that the calibration of individual sensors is unavoidable and the results are successful with errors of negligible magnitudes.

\subsection{Location tracking}

To evaluate location tracking using the tags and anchors, we walked from location to location in a clockwise pattern starting at the location of coordinate $(0,0)$. In every location, we collected at least 10 points of ranging data to the three anchors, and we established the location with a 2D trilateration algorithm based on a least-squares method. Figure 7 illustrates the trajectory of the dynamic tag. Compared with the actual movement of the tag, reported values deviated from 9.8 to $40.1 \mathrm{~cm}$. Multiple factors contribute to these deviations. One is the antenna delay that impacts the ranging values. Another factor can be the trilateration algorithm that approximates the location since it does not have correct ranging values. Although a $40 \mathrm{~cm}$ error is still acceptable in indoor localization, in our future work, we will focus on producing more accurate results. 


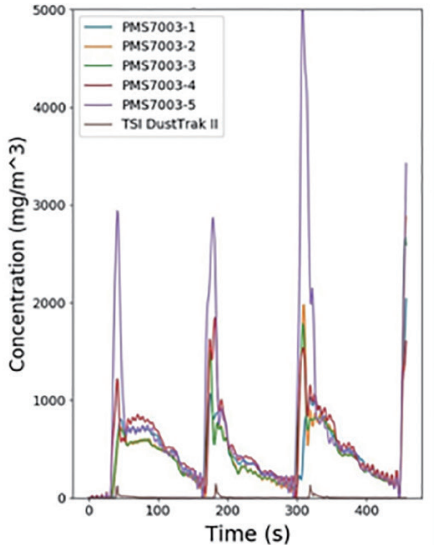

(a)

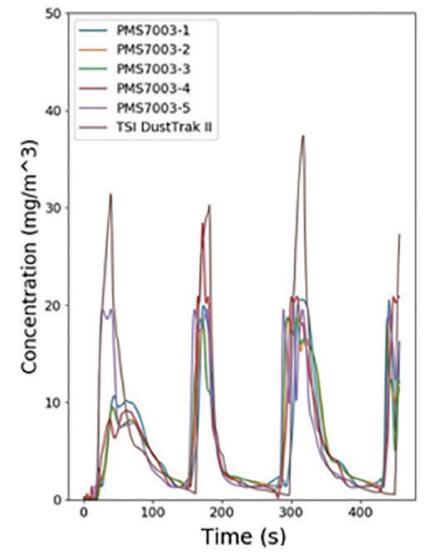

(c)

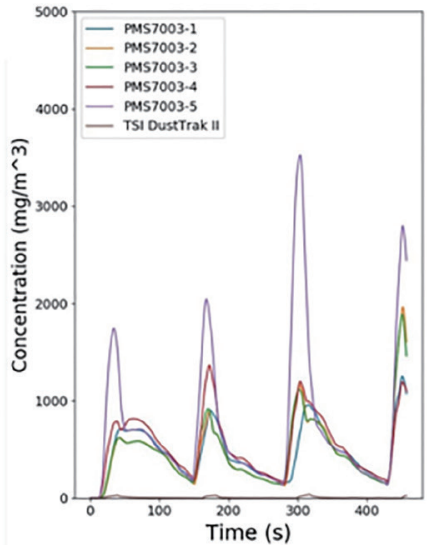

(b)

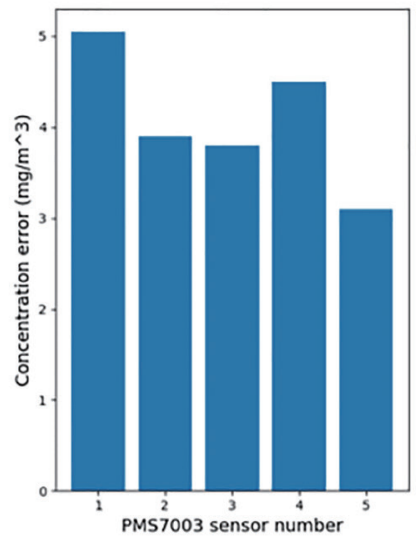

(d)

Fig. 6. (Color online) (a) Collected noisy sensor data, (b) smoothed with a moving average of window size 20, (c) calibrated using a linear fitting function, and (d) average calibration error.

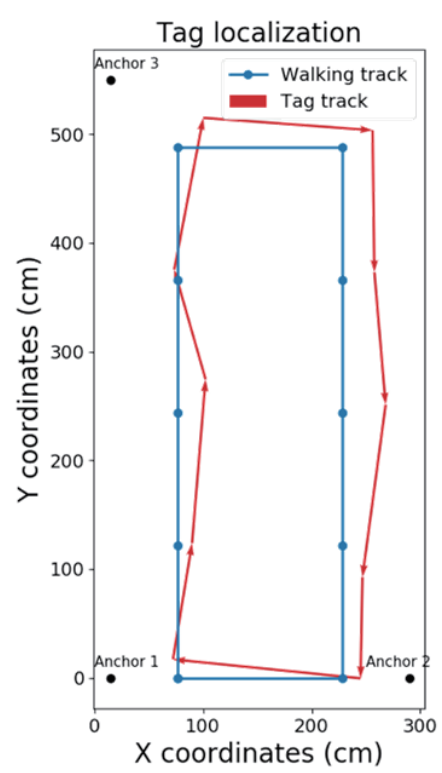

\begin{tabular}{|c|c|}
\hline Location $(\mathrm{cm})$ & Localization error $(\mathrm{cm})$ \\
\hline$(0,0)$ & 17.3 \\
\hline$(0,122)$ & 13.9 \\
\hline$(0,244)$ & 40.1 \\
\hline$(0,366)$ & 9.8 \\
\hline$(0,488)$ & 36.1 \\
\hline$(152,488)$ & 31.7 \\
\hline$(152,366)$ & 30.1 \\
\hline$(152,244)$ & 40.3 \\
\hline$(152,122)$ & 34.7 \\
\hline$(152,0)$ & 16.6 \\
\hline
\end{tabular}

Fig. 7. (Color online) Tag localization error per location as the tag moves along the designated track. 


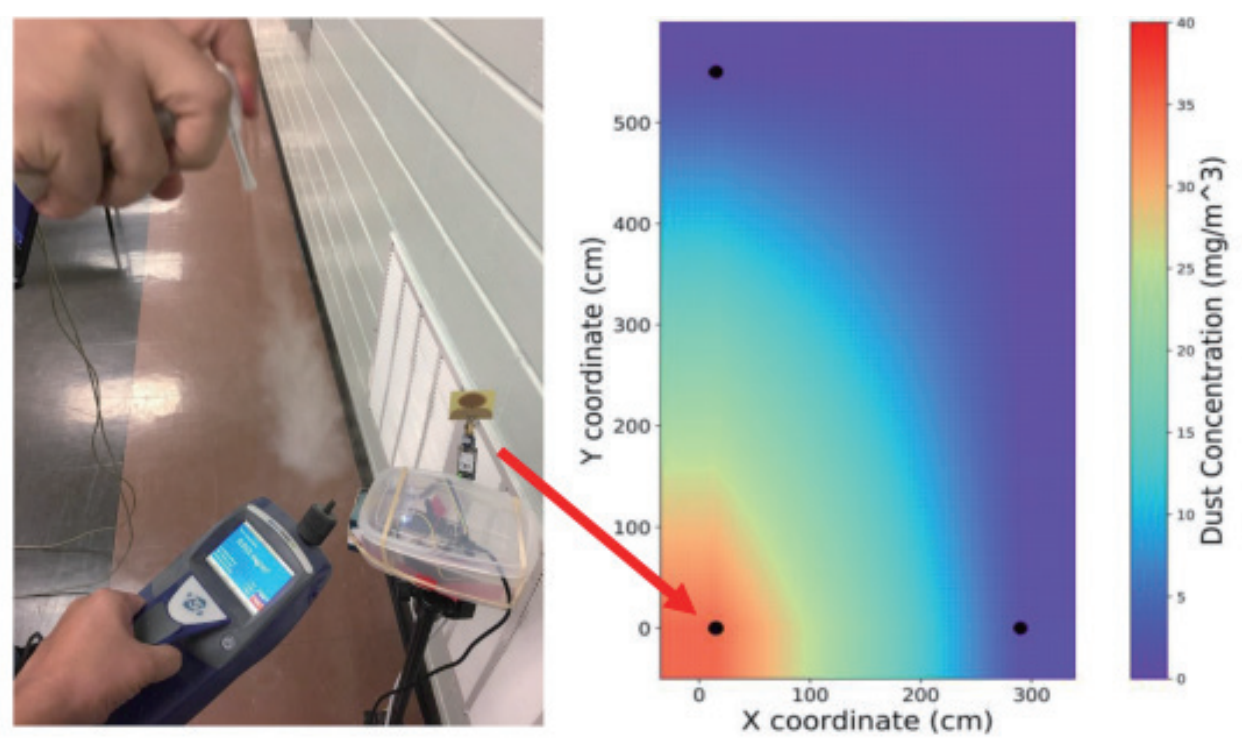

Fig. 8. (Color online) Visualization of dust levels using spatial inference demonstrating the effect of spraying dust in the vicinity of a dust sensor on the surrounding space.

\subsection{Spatial inference}

A spatial inference was used to better visualize the result of dust level monitoring. We split the experimentation space into a $10 \times 20$ grid of small locations and we used a $2 \mathrm{D}$ interpolation spline function to infer the levels of dust in every section of the grid. As shown in Fig. 8, when the dust concentration level changes in the vicinity of the sensor, we can see via different colors that the dust concentration level changes in other sectors of the experimentation space.

\section{Conclusions}

Workers are continuously exposed to the risk of inhaling toxic dust produced during construction activities. Despite the seriousness of the problem, the construction industry does not currently have an effective method of monitoring respirable dust continuously. In this research, we presented a framework and a prototype system that enables the low-cost and continuous monitoring of respirable dust in workspaces. The framework is composed of the automated calibration of low-cost sensors, deployment using location tracking sensors, and realtime visualization in a construction management platform. A test in a controlled environment demonstrated that the proposed concept has the potential to improve the current process of monitoring dust levels in large construction projects without an excessive investment. Even though this research focused on proposing a new framework, a practical implementation of the approach is possible by addressing many technical problems that were not solved by this research. First, the accuracy of dust sensor calibration can be greatly improved by incorporating other factors (e.g., temperature, humidity, and airflow) that may impact the performances after calibration. Similarly, the accuracy of location tracking still must be improved by applying 
proper location-estimating algorithms. Also, the spatial inference and visualization of dust levels should incorporate the movements of dust particles. In this research, many of these variables were not fully considered while the prototype system was being developed. Our future research will address the technical limitations identified and discussed in this report.

\section{Acknowledgments}

Wonho Suh's research was sponsored by NRF-2017R1D1A1A09000606 and the Institute for Information \& Communications Technology Promotion (IITP) grant (No. 2017-0-01031) of the Korean government. Young-Joo Lee's research was sponsored by a grant (18SCIP-B138406-03) from the Smart Civil Infrastructure Research Program funded by the Ministry of Land, Infrastructure and Transport (MOLIT) of the Korea government and the Korea Agency for Infrastructure Technology Advancement (KAIA).

\section{References}

1 OSHA: OSHA's Final Rule to Protect Workers from Exposure to Respirable Crystalline Silica: https://www. osha.gov/silica/ (accessed December 2017).

2 M. E. Flanagan, N. Seixas, P. Becker, B. Takacs, and J. Camp: J. Occup. Environ. Hyg. 3 (2006) 144.

3 L. Y. Ding, C. Zhou, Q. X. Deng, H. B. Luo, X. W. Ye, Y. Q. Ni, and P. Guo: Autom. Constr. 36 (2013) 25.

4 Z. Riaz, M. Arslan, A. K. Kiani, and S. Azhar: Automat. Constr. 45 (2014) 96.

5 S. O. Cheung, K. K. W. Cheung, and H. C. H. Suen: J. Saf. Res. 35 (2004) 159.

6 R. Williams, A. Kaufman, T. Hanley, J. Rice, and S. Garvey: Evaluation of Field-deployed Low-cost PM Sensors (Environmental Protection Agency, Washington, D.C., 2014).

7 V. H. Bennetts, E. Schaffernicht, A. J. Lilienthal, H. Fan, T. P. Kucner, L. Andersson, and A. Johansson: 2016 IEEE/RSJ Int. Conf. Intelligent Robots and Systems (IROS) (2016) 131.

8 T. Cheng, M. Venugopal, J. Teizer, and P. A. Vela: Autom. Constr. 20 (2011) 1173.

9 T. Cheng and J. Teizer: Autom. Constr. 34 (2013) 3.

10 B. Bennett, A. G. Cohn, and A. Isli: Proc. 9th IEEE Int. Conf. Tools with Artificial Intelligence (ICTAI'97) (IEEE, 1997) 314.

11 M. S. Wong, E. Mok, T. Wang, and Z. Yong: Procedia Environ. Sci. 36 (2016) 207.

12 G. Dedes and A. G. Dempster: IEEE 62nd Vehicular Technology Conf. (IEEE, 2005) 412.

13 T. Kuflik, J. Lanir, E. Dim, A. Wecker, M. Corra, M. Zancanaro, and O. Stock: Proc. 16th Int. Conf. Intelligent User Interfaces (2011) 375.

14 M.-Y. Chuang and K.-T Feng: IEEE 63rd Vehicular Technology Conf. (IEEE, 2006) 2612.

15 V. Otsason, A. Varshavsky, A. LaMarca, and E. de Lara: Int. Conf. Ubiquitous Computing (2005) 141.

16 H. Wang, S. Sen, A. Elgohary, M. Farid, M. Youssef, and R. R. Choudhury: Proc. 10th Int. Conf. Mobile Systems, Applications, and Services (2012) 197.

17 A. Rai, K. K. Chintalapudi, V. N. Padmanabhan, and R. Sen: Proc. 18th Annual Int. Conf. Mobile Computing and Networking (2012) 293.

18 F. Li, C. Zhao, G. Ding, J. Gong, C. Liu, and F. Zhao: Proc. 2012 ACM Conf. Ubiquitous Computing (2012) 421.

19 P. Lazik, N. Rajagopal, A. Rowe, and B. Sinopoli: Real-Time and Embedded Technology and Applications Symp. (RTAS) (IEEE, 2015) 108.

20 P. Lazik and A. Rowe: Proc. 10th ACM Conf. Embedded Network Sensor Systems (2012) 99.

21 G. Jin, X. Lu, and M.-S. Park: IEEE Int. Conf. Sensor Networks, Ubiquitous, and Trustworthy Computing (SUTC'06) (Taichung, 2006) 4.

22 A. Alarifi, A. Al-Salman, M. Alsaleh, A. Alnafessah, S. Al-Hadhrami, M. A. Al-Ammar, and H. S. AlKhalifa: Sensors 16 (2016) 707.

23 B. Kempke, P. Pannuto, B. Campbell, and P. Dutta: Proc. 14th ACM Conf. Embedded Network Sensor Systems (2016) 137. 
24 B. Kempke, P. Pannuto, and P. Dutta: Proc. 15th Int. Conf. Information Processing in Sensor Networks (2016) 15.

25 K. Mikhaylov, A. Tikanmäki, J. Petäjäjärvi, M. Hämäläinen, and R. Kohno: 2016 10th Int. Symp. Medical Information and Communication Technology (ISMICT) (Worcester, MA, 2016) 1.

26 K. Sulankivi, T. Mäkelä, and M. Kiviniemi: VTT Symp. (Valtion Teknillinen Tutkimuskeskus, 2009) 125.

27 R. Jongeling, J. Kim, M. Fischer, C. Mourgues, and T. Olofsson: Autom. Constr. 17 (2008) 780. 